\title{
RIESGO DE DAÑO CEREBRAL EN PREMATUROS MENORES DE 34 SEMANAS EXPUESTOS A CORIOAMNIONITIS HISTOLÓGICA, LIMA, PERÚ
}

\author{
Noelia Guillén (1]1,a, Carolina Llerena (1),a, Susan Samalvides (101,b, Judith Vila (1) 1,2,b, \\ Tania Juárez $\mathbb{1}^{2, b}$, Jaime Cáceres $\mathbb{B}^{2, c}$, Daniel Guillén-Pinto $\mathbb{1 B}^{1,2, b}$ \\ 1 Universidad Peruana Cayetano Heredia, Lima, Perú. \\ 2 Hospital Cayetano Heredia, Lima, Perú. \\ a Médica; ${ }^{\mathrm{b}}$ neuróloga/o pediatra; ${ }^{\mathrm{c}}$ patólogo
}

\section{RESUMEN}

Objetivos: Evaluar el riesgo de daño cerebral en prematuros menores de 34 semanas expuestos a corioamnionitis histológica (CAH). Materiales y métodos: Se realizó un estudio de cohortes en el Hospital Cayetano Heredia, durante el 2015. Fueron incluidos prematuros menores de 34 semanas que tuvieran examen histopatológico de la placenta. Los tipos de $\mathrm{CAH}$ evaluados fueron subcorionitis, corionitis, corioamnionitis, con o sin funisitis. El daño cerebral se evaluó en tres periodos de edad, entre 0 y 7 días, entre 7 y 30 días y a las 40 semanas gestacionales corregidas. Se realizó un seguimiento neurológico y controles con ecografía cerebral. Resultados: Se estudiaron 85 prematuros, $47,1 \%$ eran mujeres y la media de la edad gestacional fue de 30,9 semanas. El 42\% (36/85) nacieron expuestos a CAH. La ruptura prematura de membrana fue la principal generatriz de sepsis, y la sepsis se relacionó con daño neurológico. La CAH estuvo asociada con hemorragia intraventricular (HIV) durante la primera semana y con lesiones de la sustancia blanca entre los 7 y 30 días de edad $(\mathrm{p}=0,035)$. El tipo corioamnionitis de CAH se asoció al daño neurológico durante la primera semana ( $R R=2,11$; IC 95\%: 1,09-4,11) y entre los 7 y 30 días de vida ( $R R=2,72$; IC 95\%: 1,07-6,88). Conclusiones: La corioamnionitis fue un factor de riesgo para desarrollar lesiones cerebrales en prematuros menores de 34 semanas, para HIV durante los primeros 7 días y lesiones de sustancia blanca entre los 7 y los 30 días de edad. A las 40 semanas de edad corregida, los prematuros extremos con CAH tuvieron lesiones cerebrales más extensas.

Palabras Clave: Corioamnionitis Histológica; Prematuro; Hemorragia Intraventricular; Sustancia Blanca; Leucomalacia Periventricular; Vasculopatía Lenticuloestriada; Neurología; Neonatología (fuente: DeCS BIREME).

\section{RISK OF BRAIN DAMAGE IN PREMATURE INFANTS UNDER 34 WEEKS OF GESTATIONAL AGE EXPOSED TO HISTOLOGICAL CHORIOAMNIONITIS, LIMA, PERU}

Citar como: Guillén N, Llerena C, Samalvides S, Vila J, Juárez T, Cáceres J, et al. Riesgo de daño cerebral en prematuros menores de 34 semanas expuestos a corioamnionitis histológica, Lima, Perú. Rev Peru Med Exp Salud Publica. 2020;37(2):229-38 doi: https://doi.org/10.17843/ rpmesp.2020.372.4779

Correspondencia: Noelia Guillén; calle Modesto Lafuente $\mathrm{N}^{\circ}$ 57, piso 5 , puerta A, CP 28003, Madrid, España; noelia.guillen.m@gmail.com

Recibido: 03/09/2019

Aprobado: 29/04/2020

En línea: $12 / 06 / 2020$

\section{ABSTRACT}

Objectives: To assess the risk of brain damage in premature infants under 34 weeks of gestational age exposed to histological chorioamnionitis (HCA). Materials and methods: A cohort study was conducted at the Hospital Cayetano Heredia, during 2015. Premature infants under 34 weeks of gestational age, who had histopathological examination of the placenta, were included. The types of HCA evaluated were sub-chorionitis, chorionitis, chorioamnionitis, with or without funisitis. Brain damage was evaluated in three age periods, between 0 and 7 days, between 7 and 30 days and at 40 weeks of corrected gestational age. A neurological follow-up and regular controls were performed with brain ultrasound. Results: A total of 85 premature infants were included, $47.1 \%$ were women and the mean gestational age was 30.9 weeks. From the total, $42 \%$ (36/85) were born exposed to HCA. Premature rupture of membranes was the main cause of sepsis, which was related to neurological damage. HCA was associated with intraventricular hemorrhage (IVH) during the first week and with white matter lesions between 7 and 30 days of age ( $\mathrm{p}=0.035)$. The chorioamnionitis type of HCA was associated with neurological damage during the first week $(\mathrm{RR}=2.11,95 \% \mathrm{CI}: 1.09-4.11)$ and between 7 and 30 days of age $(\mathrm{RR}=2.72,95 \% \mathrm{CI}$ : 1.07-6.88). Conclusions: Chorioamnionitis was a risk factor for developing brain injuries in premature infants under 34 weeks of gestational age. It was also a risk factor for HIV during the first 7 days and for white matter injuries between 7 and 30 days of age. At 40 weeks of corrected gestational age, extreme premature infants with HCA had more extensive brain damage.

Keywords: Histological Chorioamnionitis; Preterm; Intraventricular Hemorrhage; White Matter; Periventricular Leucomalacia; Lenticulostriate Vasculopathy; Neurology; Neonatology (Source: MeSH NLM). 


\section{INTRODUCCIÓN}

Hace cinco décadas Eastman y DeLeon ${ }^{(1)}$ relacionaron la fiebre materna con la parálisis cerebral (PC) infantil en neonatos a término. Desde entonces se sabe que la corioamnionitis (CA) puede inducir el parto pretérmino y potencialmente causar daño cerebral en el neonato ${ }^{(2)}$.

La CA se produce por invasión bacteriana de la placenta con posterior infiltración de polimorfonucleares $(\mathrm{PMN})^{(3)}, \mathrm{y}$ es considerada como una de las principales causas de morbilidad obstétrica en Lima ${ }^{(4)}$. La CA clínica (CAC) afecta del $0,5 \%$ al $10 \%$ de todas las gestaciones, y aumenta tres veces el riesgo de parto prematuro ${ }^{(5)}$. Mientras, que la CA histológica (CAH) está presente en $25-40 \%$ de todos los partos pretérmino y hasta en $80 \%$ en los prematuros menores de 30 semanas ${ }^{(6)}$. Al respecto, varios estudios han demostrado la escasa correlación entre el diagnóstico clínico y el histológico ${ }^{(2,5,7)}$.

En el Perú, se han descrito las lesiones cerebrales prevalentes en prematuros menores de 34 semanas, según el examen clínico y ecográfico seriado, las que fueron de mayor grado cuanto menor fue la edad gestacional ${ }^{(8)}$. Asimismo, por estudios experimentales y observaciones clínicas, se conoce que la CA puede producir lesiones cerebrales por invasión directa de las bacterias patógenas (sepsis/meningitis) ${ }^{(9-1)} \mathrm{o}$ por efecto de los productos de la inflamación (citoquinas) que se generan durante la infección placentaria ${ }^{(6,12)}$. En este proceso, las citoquinas podrían pasar la barrera hematoencefálica (BHE), lesionar la sustancia blanca cerebral ${ }^{(6,11)} \mathrm{y}$ en ocasiones generar hemorragia intraventricular ${ }^{(2)}$.

A largo plazo, se ha observado que los neonatos expuestos a CAH tienen mayor riesgo de desarrollar PC y otros desordenes del neurodesarrollo ${ }^{(6,13)}$. Sin embargo, recientes estudios, han cuestionado estos hallazgos y plantean desarrollar nuevas metodologías de investigación que permitan resolver el posible mecanismo subyacente ${ }^{(14,15)}$.

Dado que el estudio histopatológico de la placenta y el seguimiento clínico ofrecen mayor evidencia para caracterizar la posible asociación entre $\mathrm{CAH}$ y el daño cerebral del prematuro. El objetivo de esta investigación fue explorar el riesgo de daño cerebral en prematuros menores de 34 semanas expuestos a $\mathrm{CAH}$, identificados secuencialmente por edades, hasta las 40 semanas de edad gestacional corregida. Como objetivo secundario y por su relevancia en la atención intensiva se analizaron las características maternas y neonatales según la condición de $\mathrm{CAH}$.

\section{MATERIALES Y MÉTODOS}

\section{Diseño y población}

Se realizó un estudio prospectivo de cohortes en prematuros menores de 34 semanas, en el Hospital Cayetano Heredia (Lima, Perú). Se relacionaron los subtipos de CAH con los tipos de daño cerebral por edades hasta las 40 semanas de

\section{MENSAJES CLAVE}

Motivación para realizar el estudio: La corioamnionitis es frecuente y de mucho riesgo en la gestante. No se ha determinado el potencial rol lesivo de los subtipos de corioamnionitis histológica sobre el cerebro inmaduro del prematuro.

Principales hallazgos: La infección concurrente del corion y amnios duplica el riesgo de hemorragia intraventricular durante la primera semana de vida y triplica el riesgo de lesiones de la sustancia blanca entre los 7 y los 30 días de edad en prematuros menores de 34 semanas.

Implicancias: Exhortar la vigilancia obstétrica e incluir el examen histológico de la placenta en todo parto prematuro. Recomendar la evaluación neurológica neonatal periódica.

edad gestacional corregida. El tamaño muestral se estimó en 36 prematuros sin antecedente de CAH y 36 prematuros con antecedente de CAH; para este cálculo se asumió un nivel de confianza del $95 \%$, potencia del $80 \%$ y precisión de 0,133 que es la diferencia de las probabilidades anticipadas de enfermedad neurológica entre los prematuros expuestos $(0,583)$ y no expuestos a CAH $(0,450)$, tomando como base un piloto exploratorio en el mismo escenario y bajo las mismas condiciones.

De forma consecutiva se incluyeron a los prematuros menores de 34 semanas que nacieron durante el año 2015, y que tuvieran examen histológico de placenta. La edad gestacional fue registrada en el siguiente orden de certeza, por fecha de la última menstruación, ecografía de primer trimestre o escala de Ballard. Se excluyeron a los recién nacidos con malformaciones severas y aquellos cuyas madres tuvieron patología neuropsiquiátrica no controlada.

\section{Variables}

Los datos maternos, neonatales, antropométricos, la evolución clínica, ecografías y el examen histopatológico de la placenta fueron registrados en una ficha clínica. Todas las placentas tuvieron un examen macroscópico y microscópico. La CAH se definió como la presencia de infiltrado PMN difuso o parcelar en la placenta ( $\geq 10 \mathrm{PMN} /$ campo). Los hallazgos patológicos se clasificaron según la progresión de la inflamación, y se definieron los siguientes tipos: subcorionitis, corionitis y corioamnionitis. Asimismo, se exploró la funisitis, definida por la presencia de infiltración de PMN en el cordón umbilical.

De acuerdo con la mayor probabilidad de lesiones cerebrales por edad del prematuro, el daño cerebral ${ }^{(7)}$ se definió como cualquier tipo de injuria cerebral identificable durante la primera semana ( 0 a 7 días), entre la primera y cuarta se- 
mana (7 a 30 días) y a las 40 semanas de edad corregida. Los tipos de daño se clasificaron en hemorragia intraventricular (HIV), leucomalacia periventricular (LPV), encefalopatía neonatal (EN), meningitis (MEC), vasculopatía lentículo estriada (VLE), hemorragia parenquimal (HP), infarto cerebral (IC) e hipoplasia cerebral (HC). Se consideró que las lesiones hemorrágicas e isquémicas tienden a ser mucho más frecuentes en la primera semana, las de sustancia blanca entre la tercera y la cuarta semana, y los efectos tróficos cerebrales a las 40 semanas gestacionales de edad corregida ${ }^{(8,16)}$.

La sepsis probable se consideró en aquellos casos con signos clínicos y evidencia de leucocitosis, proteína $C$ reactiva o procalcitonina positiva. La sepsis confirmada se consideró en aquellos casos con signos clínicos y hemocultivos positivos ${ }^{(17)}$.

Los casos de meningitis requirieron la demostración de pleocitosis ( $\geq 30$ leucocitos por mililitro en el LCR), los casos de encefalopatía fueron definidos por la presencia de manifestaciones clínicas (hipoactividad, hipotonía, crisis epilépticas) y acidosis metabólica.

Todos los pacientes tuvieron examen neurológico, medición del perímetro cefálico y ecografía cerebral, durante los primeros tres días, luego semanal durante tres semanas, y posteriormente cada cuatro semanas hasta las 40 semanas de edad corregida, realizadas por un especialista en neuropediatría. Todas las ecografías tuvieron una segunda revisión por un especialista en ultrasonido cerebral neonatal.

\section{Consideraciones éticas}

Todos los niños recibieron atención neonatal en las unidades de cuidados intensivos como parte del protocolo de atención del hospital y del Ministerio de Salud del Perú ${ }^{(18) .}$ En todos los casos se solicitó el consentimiento informado; además, cuando la madre era menor de 18 años se solicitó el asentimiento y consentimiento de un familiar mayor de edad. El estudio fue aprobado por los comités de Ética de la Universidad Peruana Cayetano Heredia y del Hospital Cayetano Heredia.

\section{Análisis estadístico}

La información recolectada fue almacenada en una base de datos protegida. Luego, fue analizada usando el programa STATA versión 13. Las variables numéricas con distribución normal se analizaron con la prueba $\mathrm{T}$ de Student, y las variables numéricas sin distribución normal se analizaron con la prueba de U de Mann Whitney.

Para el análisis de las características neonatales a nivel bivariado se usó la prueba exacta de Fisher y Chi cuadrado, dependiendo de las frecuencias esperadas. Para el análisis multivariado, las variables se analizaron por grupos de edad del prematuro, de 0 a 7 días, de 7 a 30 días y a las 40 semanas de edad corregida, de acuerdo con la probabilidad de mayor riesgo. El riesgo relativo de desarrollar daño ce- rebral en los neonatos expuestos a CAH se determinó con un modelo lineal generalizado, de la familia de Poisson para variables dicotómicas, usado por la forma de la distribución de la variable de respuesta (daño cerebral), con varianza de error robusta. Para este análisis, se incluyeron variables de confusión que tuvieron asociación con el daño cerebral en el análisis bivariado.

\section{RESULTADOS}

\section{Enrolamiento de pacientes}

En el año 2015 nacieron 102 prematuros menores de 34 semanas. Fueron incluidos 85 pacientes, de los cuales 66 concluyeron el seguimiento hasta las 40 semanas de edad corregida, 6 abandonaron el seguimiento (7,1\%) y 13 fallecieron (15,3\%). El estudio se cerró en marzo del 2016 al concluir el seguimiento del último prematuro (Figura 1).

\section{Características generales de la población de estudio}

El 42,4\% (36/85) de los prematuros nacieron expuestos a CAH (Figura 1). La edad materna promedio fue 27,6 años (DE: 7); y el 45,9\% (39/85) tuvieron controles prenatales adecuados. Como antecedentes maternos destacaron la in-

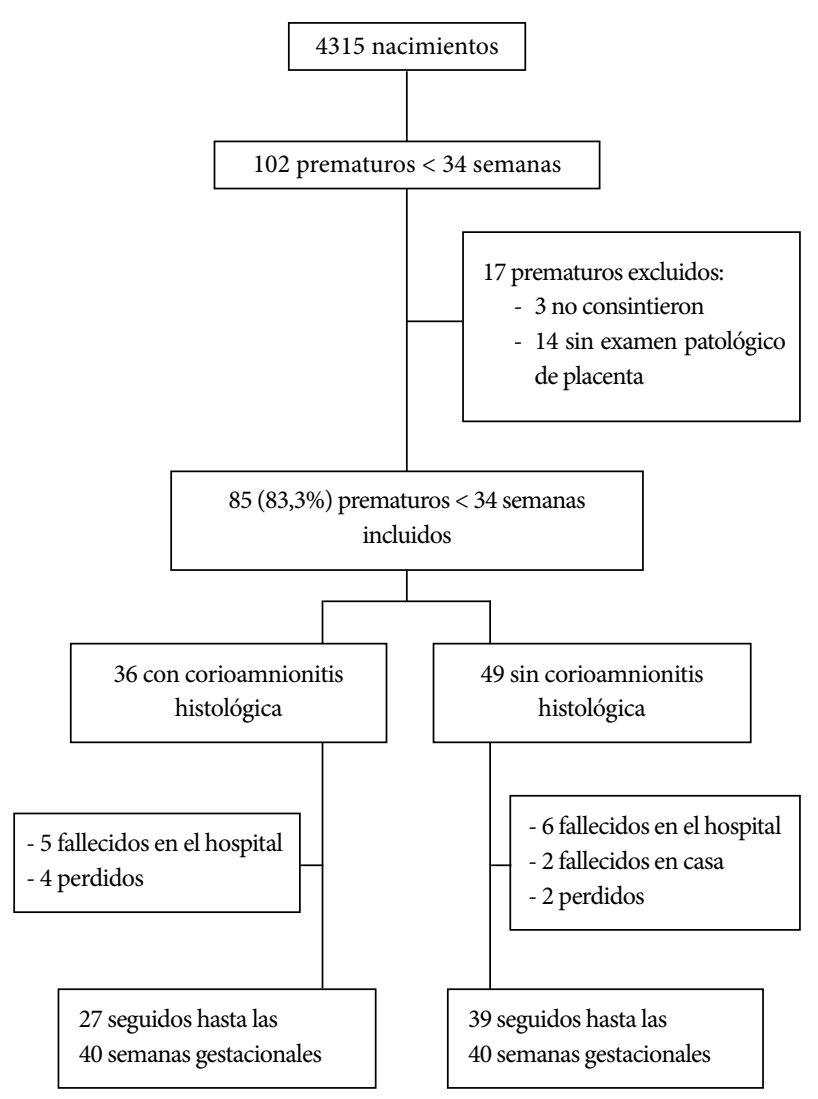

Figura 1. Flujograma de la inclusión de los pacientes. 
fección urinaria y ruptura prematura de membranas (RPM), presentes en el $28,2 \%$ y $25,9 \%$ de las madres, respectivamente. La edad gestacional fue de 30,9 semanas (DE: 2,6) y el peso promedio fue de $1670 \mathrm{~g}$ (DE: 526,1), encontrándose la mayoría con bajo peso para edad gestacional; asimismo, la talla al nacimiento fue $41,3 \mathrm{~cm}$ (DE: 4,5) (Tabla 1).

\section{Características asociadas a la corioamnionitis histológica}

Según la extensión, la CAH se distribuyó de la siguiente manera: subcorionitis 38,9\% (14/36), corionitis 38,9\% (14/36), y corioamnionitis en el 22,2\% (8/36). Todos los casos de funisitis $44,4 \%(16 / 36)$ se encontraron combinados con algún tipo de afectación en las capas placentarias.
El factor educación materna se asoció negativamente a CAH ( $\mathrm{p}=0,042)$. La RPM y la ruptura de membranas mayor de 18 horas fueron más frecuentes en los prematuros expuestos a CAH ( $p=0,004$ y $p=0,033$, respectivamente). El uso de corticoides prenatales fue menos frecuente en el grupo con CAH ( $p=0,002)$. El parto eutócico fue más frecuente en el grupo expuesto a CAH ( $\mathrm{p}=0,044)$, mientras que la cesárea sin trabajo de parto, en el grupo no expuesto $(\mathrm{p}=0,044)$. Otras características de la madre, según el estado de $\mathrm{CAH}$, se detallan en la Tabla 2.

El promedio del percentil del perímetro cefálico de nacimiento del grupo con CAH fue de 47,6 (desviación estándar: 28,3), menor $(\mathrm{p}=0,035)$ que en el grupo sin $\mathrm{CAH}$

Tabla 1. Frecuencia de las características maternas y neonatales

\begin{tabular}{|c|c|c|c|}
\hline Maternas & n (\%) & Neonatales & n (\%) \\
\hline Edad, media (DE) & $29(4,6)$ & Sexo & \\
\hline Grado de instrucción & & Femenino & $40(47,1)$ \\
\hline Primaria & $4(4,7)$ & Masculino & $45(52,9)$ \\
\hline Secundaria & $58(68,2)$ & Edad gestacional (semanas) & \\
\hline Superior & $23(27,1)$ & 24 a 29 & $26(30,6)$ \\
\hline Estado civil & & 30 a 31 & $27(31,8)$ \\
\hline Soltera & $20(23,5)$ & 32 a 33 & $32(37,6)$ \\
\hline Conviviente & $52(61,2)$ & Peso, media (DE) & $1670,5(526)$ \\
\hline Casada & $13(15,3)$ & Talla, media (DE) & $41,3(4,5)$ \\
\hline Controles prenatales adecuados & $39(45,9)$ & Perímetro cefálico al nacer, media (DE) & $28,7(2,8)$ \\
\hline Corioamnionitis clínica & $6(7,1)$ & Percentil del perímetro cefálico, media (DE) & $55,2(28,5)$ \\
\hline Gestación múltiple & $18(21,2)$ & Adecuación peso/edad gestacional & \\
\hline Vaginosis & $13(15,3)$ & Adecuado & $2(2,4)$ \\
\hline Infección del tracto urinario & $24(28,2)$ & Bajo peso al nacer & $56(65,9)$ \\
\hline Hipertensión gestacional & $6(7,1)$ & Muy bajo peso al nacer & $16(18,8)$ \\
\hline Preeclampsia / eclampsia & $15(17,6)$ & Extremo bajo peso al nacer & $11(12,9)$ \\
\hline Hemorragia del tercer trimestre & $12(14,1)$ & Fallecidos & $13(15,3)$ \\
\hline Retardo del crecimiento intrauterino & $6(7,1)$ & Sepsis & \\
\hline Diabetes gestacional & $6(7,1)$ & Ausente & $32(37,6)$ \\
\hline Fiebre periparto & $15(17,6)$ & Posible & $4(4,7)$ \\
\hline Ruptura prematura de membranas pretérmino & $22(25,9)$ & Probable & $28(32,9)$ \\
\hline Ruptura prematura de membranas $>18$ horas & $13(15,3)$ & Confirmada & $21(24,7)$ \\
\hline Ecografía obstétrica anormal & $10(11,8)$ & Insuficiencia renal aguda & $12(14,1)$ \\
\hline Uso de corticoides prenatales & $27(31,8)$ & Enfermedad de membrana hialina & $45(52,9)$ \\
\hline Parto & & Hipoglicemia & $21(24,7)$ \\
\hline Eutócico & $19(22,4)$ & Neumonía & $14(16,5)$ \\
\hline Cesárea con trabajo de parto & $23(27,1)$ & Anemia & $29(34,1)$ \\
\hline Cesárea sin trabajo de parto & $43(50,6)$ & Apnea de la prematuridad & $24(28,2)$ \\
\hline Reanimación & & Enterocolitis necrotizante & $6(7,1)$ \\
\hline No & $25(29,4)$ & Ictericia & $49(57,6)$ \\
\hline Oxígeno & $7(8,2)$ & Encefalopatía neonatal & $9(10,6)$ \\
\hline Neopuff & $39(45,9)$ & Encefalopatía por bilirrubina & $1(1,2)$ \\
\hline Tubo en $\mathrm{T}$ & $8(9,4)$ & Persistencia ductus arterioso & $22(25,9)$ \\
\hline Masaje & $6(7,1)$ & Neumotórax & $5(5,9)$ \\
\hline \multicolumn{4}{|l|}{ Líquido amniótico } \\
\hline Claro & $74(87,1)$ & & \\
\hline Meconial fluido & $8(9,4)$ & & \\
\hline Meconial espeso & $3(3,5)$ & & \\
\hline
\end{tabular}


Tabla 2. Características maternas según la condición de corioamnionitis histológica

\begin{tabular}{|c|c|c|c|}
\hline \multirow[t]{2}{*}{ Características maternas } & \multicolumn{2}{|c|}{$\begin{array}{l}\text { Corioamnionitis } \\
\text { histológica }\end{array}$} & \multirow{2}{*}{$\begin{array}{c}\text { Valor } \\
\text { dep }\end{array}$} \\
\hline & No $(n=49)$ & Sí (n= 36) & \\
\hline Edad materna, media (DE) & $28,2(6,6)$ & $26,8(7,5)$ & $0,389^{a}$ \\
\hline Grado de instrucción & & & $0,042^{b}$ \\
\hline Primaria & $4(8,1)$ & $0(0,0)$ & \\
\hline Secundaria & $36(73,5)$ & $22(61,1)$ & \\
\hline Superior & $9(18,4)$ & $14(38,9)$ & \\
\hline Estado civil & & & $0,425^{\mathrm{c}}$ \\
\hline Soltera & $9(18,4)$ & $11(30,5)$ & \\
\hline Conviviente & $32(65,3)$ & $20(55,6)$ & \\
\hline Casada & $8(16,3)$ & $5(13,9)$ & \\
\hline Controles prenatales adecuados & $25(51,0)$ & $14(38,9)$ & $0,267^{\mathrm{c}}$ \\
\hline Corioamnionitis clínica & $2(4,1)$ & $4(11,1)$ & $0,394^{\mathrm{b}}$ \\
\hline Gestación múltiple & $15(30,6)$ & $3(8,3)$ & $0,013^{\mathrm{c}}$ \\
\hline Vaginosis & $7(14,3)$ & $6(16,7)$ & $0,763^{\mathrm{c}}$ \\
\hline Infección del tracto urinario & $13(26,5)$ & $11(30,6)$ & $0,684^{\mathrm{c}}$ \\
\hline Hipertensión gestacional & $3(6,1)$ & $3(8,3)$ & $0,695^{\mathrm{b}}$ \\
\hline Preeclampsia / eclampsia & $11(22,5)$ & $4(11,1)$ & $0,175^{\mathrm{c}}$ \\
\hline Hemorragia del tercer trimestre & $9(18,4)$ & $3(8,3)$ & $0,189^{\mathrm{c}}$ \\
\hline Retardo del crecimiento intrautero & $6(12,2)$ & $0(0,0)$ & $0,036^{\mathrm{b}}$ \\
\hline Diabetes gestacional & $3(6,1)$ & $3(8,3)$ & $0,695^{b}$ \\
\hline Fiebre periparto & $7(14,3)$ & $8(22,2)$ & $0,343^{\mathrm{c}}$ \\
\hline $\begin{array}{l}\text { Ruptura prematura de membranas } \\
\text { pretérmino }\end{array}$ & $7(14,3)$ & $15(41,7)$ & $0,004^{\mathrm{c}}$ \\
\hline $\begin{array}{l}\text { Ruptura prematura de membranas } \\
>18 \mathrm{~h}\end{array}$ & $4(8,2)$ & $9(25,0)$ & $0,033^{\mathrm{c}}$ \\
\hline Ecografía obstétrica anormal & $7(14,3)$ & $3(8,3)$ & $0,507^{\mathrm{b}}$ \\
\hline Uso de corticoides prenatales & $22(44,9)$ & $5(13,9)$ & $0,002^{\mathrm{c}}$ \\
\hline Parto & & & $0,044^{\mathrm{c}}$ \\
\hline Eutócico & $7(14,3)$ & $12(33,3)$ & \\
\hline Cesárea con trabajo de parto & $12(24,5)$ & $11(30,6)$ & \\
\hline Cesárea sin trabajo de parto & $30(61,2)$ & $13(36,1)$ & \\
\hline Reanimación & & & $0,316^{\mathrm{b}}$ \\
\hline No & $14(28,6)$ & $11(30,6)$ & \\
\hline Oxígeno & $5(10,2)$ & $2(5,6)$ & \\
\hline Neopuff & $25(51,0)$ & $14(38,9)$ & \\
\hline Tubo en $\mathrm{T}$ & $2(4,1)$ & $6(16,7)$ & \\
\hline Masaje & $3(6,1)$ & $3(8,3)$ & \\
\hline Líquido amniótico & & & $0,296^{\mathrm{b}}$ \\
\hline Claro & $45(91,8)$ & $29(80,6)$ & \\
\hline Meconial fluido & $3(6,1)$ & $5(13,9)$ & \\
\hline Meconial espeso & $1(2,1)$ & $2(5,6)$ & \\
\hline
\end{tabular}

${ }^{a}$ Prueba T de Student, ${ }^{b}$ prueba exacta de Fisher, ${ }^{c}$ prueba de Chi cuadrado

(60,7, desviación estándar: 27,6). El 57,6\% (49/85) de los pacientes presentaron sepsis probable o sepsis confirmada, cuya frecuencia fue mayor en prematuros expuestos a $\mathrm{CAH}$ $(\mathrm{p}=0,013)$. Otras características del recién nacido, según estado de CAH, se describen en la Tabla 3.

\section{Características neonatales asociadas al daño neurológico}

Se encontró una gran frecuencia de patología neurológica $52,9 \%(45 / 85)$, mayor en el grupo con CAH $(63,9 \%)$ frente al grupo sin CAH $(44,9 \%)(\mathrm{p}=0,083)$. Destacando la VLE con $33,3 \%$ de casos en el grupo con CAH frente al 20,4\% en el grupo sin CAH $(p=0,179)$, y HIV con $25 \%$ en el gru-

Tabla 3. Características neonatales de los prematuros según la condición de corioamnionitis histológica

\begin{tabular}{|c|c|c|c|}
\hline \multirow[t]{2}{*}{ Características neonatales } & \multicolumn{2}{|c|}{$\begin{array}{c}\text { Corioamnionitis } \\
\text { histológica }\end{array}$} & \multirow{2}{*}{$\begin{array}{l}\text { Valor } \\
\text { de p }\end{array}$} \\
\hline & No $(n=49)$ & Sí $(n=36)$ & \\
\hline Sexo & & & $0,679^{\mathrm{a}}$ \\
\hline Femenino & $24(49,0)$ & $16(44,4)$ & \\
\hline Masculino & $25(51,0)$ & $20(55,6)$ & \\
\hline Edad gestacional & & & $0,097^{\mathrm{a}}$ \\
\hline 24 a 29 & $14(28,6)$ & $12(33,3)$ & \\
\hline 30 a 31 & $20(40,8)$ & $7(19,4)$ & \\
\hline 32 a 33 & $15(30,6)$ & $17(47,2)$ & \\
\hline Peso & $1688,7(500)$ & $1645,6(567)$ & $0,711^{\mathrm{b}}$ \\
\hline Talla, mediana (RIC) & $41,6(4,6)$ & $40,8(4,3)$ & $0,215^{\mathrm{c}}$ \\
\hline $\begin{array}{l}\text { Perímetro cefálico al nacer, } \\
\text { media (DE) }\end{array}$ & $29,1(2,3)$ & $28,2(3,3)$ & $0,179^{\mathrm{b}}$ \\
\hline $\begin{array}{l}\text { Percentil del perímetro } \\
\text { cefálico, media (DE) }\end{array}$ & $60,7(27,7)$ & $47,6(28,3)$ & $0,035^{b}$ \\
\hline \multicolumn{3}{|c|}{ Adecuación del peso / edad gestacional } & $0,818^{\mathrm{d}}$ \\
\hline Adecuado & $1(2,0)$ & $1(2,8)$ & \\
\hline Bajo peso al nacer & $34(69,4)$ & $22(61,1)$ & \\
\hline Muy bajo peso al nacer & $9(18,4)$ & $7(19,4)$ & \\
\hline Extremo bajo peso al nacer & $5(10,2)$ & $6(16,7)$ & \\
\hline Fallecidos & $8(16,3)$ & $5(13,9)$ & $0,758^{\mathrm{a}}$ \\
\hline Sepsis & & & $0,013^{\mathrm{d}}$ \\
\hline Ausente & $25(51,1)$ & $7(19,4)$ & \\
\hline Posible & $1(2,0)$ & $3(8,3)$ & \\
\hline Probable & $12(24,5)$ & $16(44,4)$ & \\
\hline Confirmada & $11(22,4)$ & $10(27,8)$ & \\
\hline Insuficiencia renal aguda & $6(12,2)$ & $6(16,7)$ & $0,563^{\mathrm{a}}$ \\
\hline $\begin{array}{l}\text { Enfermedad de membrana } \\
\text { hialina }\end{array}$ & $29(59,2)$ & $16(44,4)$ & $0,179^{\mathrm{a}}$ \\
\hline Hipoglicemia & $15(30,6)$ & $6(16,7)$ & $0,141^{\mathrm{a}}$ \\
\hline Neumonía & $8(16,3)$ & $6(16,7)$ & $0,967^{\mathrm{a}}$ \\
\hline Anemia & $16(32,7)$ & $13(36,1)$ & $0,740^{\mathrm{a}}$ \\
\hline Apnea de la prematuridad & $12(24,5)$ & $12(33,3)$ & $0,371^{\mathrm{a}}$ \\
\hline Enterocolitis necrotizante & $4(8,2)$ & $2(5,6)$ & $1,000^{\mathrm{d}}$ \\
\hline Ictericia & $26(53,1)$ & $23(63,9)$ & $0,318^{a}$ \\
\hline Encefalopatía neonatal & $5(10,2)$ & $4(11,1)$ & $1,000^{\mathrm{d}}$ \\
\hline $\begin{array}{l}\text { Encefalopatía por } \\
\text { bilirrubinas }\end{array}$ & $0(0,0)$ & $1(2,8)$ & $0,424^{\mathrm{d}}$ \\
\hline $\begin{array}{l}\text { Persistencia del conducto } \\
\text { arterioso }\end{array}$ & $12(2,5)$ & $10(27,8)$ & $0,732^{a}$ \\
\hline Neumotórax & $2(4,1)$ & $3(8,3)$ & $0,646^{\mathrm{d}}$ \\
\hline
\end{tabular}

DE: desviación estandar, RIC: rango intercuartilico

${ }^{\text {a }}$ Prueba de Chi cuadrado, ${ }^{\mathrm{b}}$ prueba $\mathrm{T}$ de Student, ${ }^{\mathrm{c}}$ prueba U de Mann-Whitney, prueba exacta de Fisher 
po con CAH comparado con $20,4 \%$ en el grupo sin CAH $(p=0,616)$. Las frecuencias de LPV, MEC y EN fueron muy pequeñas y similares en ambos grupos.

La sepsis tuvo mayor impacto sobre el daño neurológico durante la primera semana de vida $(\mathrm{p}=0,015)$ y entre 7 y 30 días de vida $(p=0,008)$. La insuficiencia renal, la enfermedad de membrana hialina, la enterocolitis necrotizante y la persistencia de conducto arterioso se encontraron asociadas al daño neurológico entre 7 y 30 días de vida $(\mathrm{p}<0,050)$. La neumonía solo tuvo impacto durante los primeros 7 días $(p=0,017)$, y la enterocolitis necrotizante fue la única asociada a alteraciones encontradas a las 40 semanas de edad corregida $(\mathrm{p}=0,029)$. La asociación entre otras patologías y el daño neurológico se presentan en la Tabla 4.

Trece prematuros fallecieron con edad gestacional promedio de 27,8 (DE: 3,5) semanas, el 61,5\% (8/13) fueron varones, y cinco presentaron corioamnionitis clínica. En el grupo sin CAH fallecieron 8 niños, de los cuales 5 presenta- ron daño neurológico 5/8 (62,5\%), y en el grupo con CAH fallecieron 5 niños, todos con daño neurológico 5/5 (100\%) (Figura 1).

\section{Riesgo de daño cerebral según antecedente de corioamnionitis histológica}

En la Tabla 5 se muestra el análisis multivariado del riesgo relativo (RR), donde se controló el efecto de las variables sepsis, uso de corticoides, insuficiencia renal aguda, neumonía, enterocolitis necrotizante y persistencia del conducto arterioso. Asimismo, se filtraron los efectos de la HIV, MEC y EN ocurridos entre el nacimiento y las cuatro primeras semanas de edad, por su potencial efecto deletéreo hasta las 40 semanas de edad gestacional.

La corioamnionitis (afectación histológica de corion y amnios) fue el único tipo de $\mathrm{CAH}$ asociado con daño neurológico $(\mathrm{p}=0,006)$. Este subtipo de $\mathrm{CAH}$, duplicó el riesgo de daño cerebral en la primera semana de vida $(\mathrm{RR}=2,11$; IC 95\%: 1,09-4,11), que incluyó HIV, EN, MEC y VLE. El

Tabla 4. Daño neurológico de acuerdo con las características clínicas neonatales por grupos de edad en prematuros menores de 34 semanas

\begin{tabular}{|c|c|c|c|c|c|c|c|c|c|}
\hline \multirow[b]{2}{*}{ Características } & \multicolumn{3}{|c|}{$\begin{array}{l}\text { De } 0 \text { a } 7 \text { días de nacido } \\
\text { (HIV, VLE, MEC, EN) }\end{array}$} & \multicolumn{3}{|c|}{$\begin{array}{c}\text { De } 7 \text { a } 30 \text { días de nacido } \\
\text { (LPV, VLE) }\end{array}$} & \multicolumn{3}{|c|}{$\begin{array}{l}\text { A las } 40 \text { semanas corregidas } \\
\text { (VLE o hipoplasia) }\end{array}$} \\
\hline & No (62) & Sí (23) & $\begin{array}{l}\text { Valor } \\
\text { de p }\end{array}$ & No $(55)$ & Sí (30) & $\begin{array}{l}\text { Valor } \\
\text { de p }\end{array}$ & No $(45)$ & Sí (21) & $\begin{array}{l}\text { Valor } \\
\text { de p }\end{array}$ \\
\hline Depresión al nacer & $2(3,2)$ & $2(8,7)$ & $0,295^{a}$ & $1(1,8)$ & $3(10,0)$ & $0,124^{\mathrm{a}}$ & $2(4,4)$ & $1(4,8)$ & $1,000^{\mathrm{a}}$ \\
\hline Edad gestacional (semanas) & & & $0,027^{b}$ & & & $0,001^{\mathrm{b}}$ & & & $0,222^{\mathrm{b}}$ \\
\hline 24 a 29 & $14(22,6)$ & $12(52,2)$ & & $10(18,2)$ & $16(53,3)$ & & $8(17,8)$ & $7(33,3)$ & \\
\hline 30 a 31 & $21(33,9)$ & $6(26,1)$ & & $18(32,7)$ & $9(30,0)$ & & $15(33,3)$ & $8(38,1)$ & \\
\hline 32 a 33 & $27(43,6)$ & $5(21,7)$ & & $27(49,1)$ & $5(17,7)$ & & $22(48,9)$ & $6(28,6)$ & \\
\hline Sepsis & & & $0,015^{a}$ & & & $0,008^{\mathrm{a}}$ & & & $0,710^{\mathrm{a}}$ \\
\hline Ausente & $28(45,2)$ & $4(17,4)$ & & $27(49,1)$ & $5(16,7)$ & & $21(46,7)$ & $7(33,3)$ & \\
\hline Posible & $3(4,8)$ & $1(4,4)$ & & $3(5,4)$ & $1(3,3)$ & & $2(4,4)$ & $2(9,5)$ & \\
\hline Probable & $21(33,9)$ & $7(30,4)$ & & $16(29,1)$ & $12(40,0)$ & & $14(31,1)$ & $8(38,1)$ & \\
\hline Confirmada & $10(16,1)$ & $11(47,8)$ & & $9(16,4)$ & $12(40,0)$ & & $8(17,8)$ & $4(19,1)$ & \\
\hline Insuficiencia renal aguda & $2(3,2)$ & $10(43,5)$ & $0,000^{\mathrm{a}}$ & $4(7,3)$ & $8(26,7)$ & $0,022^{\mathrm{a}}$ & $2(4,4)$ & $2(9,5)$ & $0,587^{\mathrm{a}}$ \\
\hline Enfermedad de membrana hialina & $28(45,2)$ & $17(73,9)$ & $0,018^{\mathrm{b}}$ & $22(40,0)$ & $23(76,7)$ & $0,001^{\mathrm{b}}$ & $18(40,0)$ & $12(57,1)$ & $0,289^{\mathrm{b}}$ \\
\hline Hipoglicemia & $14(22,6)$ & $7(30,4)$ & $0,456^{\mathrm{b}}$ & $13(23,6)$ & $8(26,7)$ & $0,757^{\mathrm{b}}$ & $9(20,0)$ & $7(33,3)$ & $0,239^{\mathrm{b}}$ \\
\hline Neumonía & $6(9,7)$ & $8(34,8)$ & $0,017^{\mathrm{a}}$ & $7(12,7)$ & $7(23,3)$ & $0,232^{\mathrm{a}}$ & $3(6,7)$ & $5(23,8)$ & $0,098^{\mathrm{a}}$ \\
\hline Anemia & $18(29,0)$ & $11(47,8)$ & $0,104^{\mathrm{b}}$ & $16(29,1)$ & $13(43,3)$ & $0,186^{\mathrm{b}}$ & $11(24,4)$ & $5(23,8)$ & $0,955^{\mathrm{b}}$ \\
\hline Apneas de la prematuridad & $19(30,7)$ & $5(21,7)$ & $0,418^{b}$ & $18(32,7)$ & $6(20,0)$ & $0,213^{\mathrm{b}}$ & $13(28,9)$ & $5(23,8)$ & $0,666^{\mathrm{b}}$ \\
\hline Enterocolitis necrotizante & $2(3,2)$ & $4(17,4)$ & $0,043^{a}$ & $0(0,0)$ & $6(20,0)$ & $0,001^{b}$ & $0(0,0)$ & $3(14,3)$ & $0,029^{\mathrm{a}}$ \\
\hline Ictericia & $36(58,1)$ & $13(56,5)$ & $0,898^{b}$ & $30(54,5)$ & $19(63,3)$ & $0,433^{b}$ & $24(53,3)$ & $15(71,4)$ & $0,164^{\mathrm{b}}$ \\
\hline Encefalopatía neonatal & $5(8,1)$ & $4(17,4)$ & $0,245^{a}$ & $6(10,9)$ & $3(10,0)$ & $1,000^{\mathrm{a}}$ & $6(13,3)$ & $1(4,8)$ & $0,416^{\mathrm{a}}$ \\
\hline Encefalopatía por bilirrubinas & $1(1,6)$ & $0(0,0)$ & $1,000^{\mathrm{a}}$ & $0(0,0)$ & $1(3,3)$ & $0,353^{\mathrm{a}}$ & $0(0,0)$ & $1(4,8)$ & $0,318^{\mathrm{a}}$ \\
\hline Persistencia del conducto arterioso & $11(17,7)$ & $11(47,8)$ & $0,005^{b}$ & $7(12,7)$ & $15(50,0)$ & $0,000^{\mathrm{b}}$ & $7(15,6)$ & $7(33,3)$ & $0,117^{\mathrm{a}}$ \\
\hline Neumotórax & $3(4,8)$ & $2(8,7)$ & $0,609^{\mathrm{a}}$ & $3(5,4)$ & $2(6,7)$ & $1,000^{\mathrm{b}}$ & $2(4,4)$ & $1(4,8)$ & $1,000^{\mathrm{a}}$ \\
\hline
\end{tabular}

HIV: hemorragia intraventricular, VLE: vasculopatía lentículo estriada, MEC: meningitis, EN: encefalopatía neonatal, LPV: leucomalacia periventricular, HC: hipoplasia cerebral

${ }^{a}$ Prueba exacta de Fisher, ${ }^{\text {b }}$ prueba de Chi cuadrado 
Tabla 5. Riesgo de daño cerebral en prematuros menores de 34 semanas expuestos a corioamnionitis histológica

\begin{tabular}{|c|c|c|c|c|c|c|c|c|c|}
\hline \multirow{2}{*}{ Hallazgos patológicos } & \multicolumn{3}{|c|}{$\begin{array}{c}\text { Entre } 0 \text { a } 7 \text { días de nacido } \\
(\mathrm{HIV}, \mathrm{VLE}, \mathrm{MEC}, \mathrm{EN}), \mathrm{n}=85\end{array}$} & \multicolumn{3}{|c|}{$\begin{array}{c}\text { Entre } 7 \text { a } 30 \text { días de nacido } \\
\text { (LPV, VLE), } \mathbf{n}=85\end{array}$} & \multicolumn{3}{|c|}{$\begin{array}{l}\text { A las } 40 \text { semanas corregidas } \\
(\mathrm{VLE}, \mathrm{HC}), \mathrm{n}=66^{\mathrm{a}}\end{array}$} \\
\hline & $\mathbf{R R}$ & $\begin{array}{l}\text { Valor } \\
\text { de p }\end{array}$ & IC 95\% & $\mathbf{R R}$ & $\begin{array}{l}\text { Valor } \\
\text { de p }\end{array}$ & IC 95\% & $\mathbf{R R}$ & $\begin{array}{l}\text { Valor } \\
\text { de p }\end{array}$ & IC 95\% \\
\hline Corioamnionitis histológica & 1,13 & 0,717 & $0,58-2,19$ & 1,43 & 0,366 & $0,66-3,10$ & 0,91 & 0,796 & $0,44-1,89$ \\
\hline Subcorionitis & 0,60 & 0,389 & $0,19-1,92$ & 1,69 & 0,189 & $0,77-3,72$ & 0,93 & 0,868 & $0,39-2,23$ \\
\hline Corioamnionitis & 2,11 & 0,027 & $1,09-4,11$ & 2,72 & 0,035 & $1,07-6,88$ & 0,83 & 0,781 & $0,22-3,08$ \\
\hline Funisitis & 1,17 & 0,616 & $0,63-2,18$ & 0,91 & 0,845 & $0,37-2,25$ & 0,80 & 0,660 & $0,30-2,16$ \\
\hline Corioamnionitis y funisitis & 2,33 & 0,042 & $1,03-5,27$ & 3,23 & 0,000 & $1,84-5,66$ & 2,66 & 0,012 & $1,24-5,71$ \\
\hline
\end{tabular}

La tabla presenta el análisis de regresión de cada una de las seis variables de forma independiente, cada fila es un análisis de regresión ajustado (Corioamnionitis histológica, Subcorionitis, Corionitis, Corioamnionitis, Funisitis, Corioamnionitis y funisitis)

HIV: hemorragia intraventricular, VLE: vasculopatía lentículo estriada, MEC: meningitis, EN: encefalopatía neonatal, LPV: leucomalacia periventricular HC: hipoplasia cerebral, RR: riesgo relativo, IC 95\%: intervalo de confianza al 95\%

a A las 40 semanas corregidas se filtró el efecto de HIV, MEC, LPV y EN.

análisis individualizado solo para HIV en el mismo periodo elevó el riesgo de daño ( $\mathrm{p}=0,007)(\mathrm{RR}=2,46$; IC 95\%: 1,28-4,72). Con el mismo factor casi se triplicó el riesgo de daño entre los 7 y los 30 días de edad, periodo que predominaron los tipos de lesión de sustancia blanca, VLE y LPV (RR = 2,72; IC 95\%: 1,07-6,88).

La funisitis como factor único no se relacionó con daño neurológico; no obstante, se observa un efecto potenciador cuando se la asocia a la corioamnionitis, pues el riesgo relativo aumenta de 2,11 a 2,33 de 0 a 7 días de edad y de 2,72 a 3,23 de 7 a 30 días de edad.

\section{Otros hallazgos relevantes}

El daño cerebral ocurrido entre los 7 y 30 días de edad se asoció con la menor edad gestacional y con el menor perímetro cefálico al mes de nacido $(\mathrm{p}=0,000)$. Asimismo, los prematuros extremos con $\mathrm{CAH}$ tuvieron lesiones cerebrales más extensas a las 40 semanas de edad corregida $(\mathrm{p}=0,027)$.

Entre los factores no placentarios, la neumonía constituye un factor de riesgo de daño neurológico en cualquier momento ( $\mathrm{p}<0,001)(\mathrm{RR}=1,89$; IC 95\% 1,34-2,69), similar a la corioamnionitis. Entre otros efectos de la corioamnionitis se encontró como factor de riesgo para la persistencia de ductus arterioso $(\mathrm{p}=0,013)(\mathrm{RR}=2,8$; IC 95\% 1,4- 5,6).

\section{DISCUSIÓN}

La corioamnionitis histológica tiene efecto deletéreo a diferentes edades del prematuro menor de 34 semanas, con riesgo de producir o contribuir con algunas lesiones cerebrales tempranas y tardías. Estos efectos son conocidos como «lesivos a distancia» porque hacen énfasis en los mecanismos mediados por citoquinas ${ }^{(12)}$.

El modelo de investigación que presentamos es innovador, porque permite relacionar el efecto de la CAH sobre el tejido cerebral visto por edades del prematuro; el diseño poco utilizado en la literatura, destaca la importancia del examen histológico de la placenta y su relación con el monitoreo neurológico y ecográfico del paciente ${ }^{(16)}$.

El daño neurológico relacionado con la corioamnionitis histológica en prematuros es un tema controversial que ha sido analizado en estudios experimentales en animales. Si bien se ha demostrado la susceptibilidad del tejido cerebral ${ }^{(19)}$, los resultados de los estudios clínicos no han podido aclarar de manera contundente esta asociación ${ }^{(15,20,21)}$.

La CA es un proceso inflamatorio intenso, cuyo mecanismo principal se inicia con la ruptura prematura de las membranas amnióticas, la que facilita el ingreso de los microorganismos de la flora vaginal al espacio intrauterino ${ }^{(3)}$. De manera que, frecuentemente, se encuentran asociadas la RPM y la ruptura prolongada de membranas ${ }^{(22)}$. Aunque los gérmenes más comunes son de la flora vaginal ${ }^{(12,20)}$, tanto como $71,1 \%$ de cultivos positivos ${ }^{(20)}$, con frecuencia hay corioamnionitis estériles, inducidas en condiciones de estrés, daño o muerte celular, como puede ser el desprendimiento de placenta o infecciones adicionales ${ }^{(3,20,23)}$.

Independientemente del riesgo bacteriológico, tanto en la madre como en el feto se desencadena una respuesta inmunológica de gran potencia. En la madre, se activa la producción de prostaglandinas y citoquinas (IL-1 $\beta$, IL-6, IL-8, TNF- $\alpha$ ) que inducen la labor de parto prematura ${ }^{(6,13)}$, y en el feto, se origina el síndrome de respuesta inflamatoria sistémica, que se correlaciona con incrementos de IL-6 en sangre de cordón ${ }^{(5,19)}$ y con el incremento de MMP-8 en el líquido amniótico ${ }^{(3,20,24)}$. En el contexto inflamatorio, ya se ha informado que el uso de esteroides en el periodo prenatal podría reducir varios efectos posnatales ${ }^{(25)}$. Tomamos con reparo el posible efecto beneficioso que tendería a una menor frecuencia de $\mathrm{CAH}$, para ser analizado en el futuro. Sin embargo, está en discusión su efecto en la injuria cerebral por CA, sobre la frecuencia de parálisis cerebral ${ }^{(21)}$, y los potenciales efectos deletéreos para el neurodesarrollo ${ }^{(26)}$. 
En el tejido cerebral, las citoquinas tienen la capacidad de alterar la permeabilidad de la BHE y producir disrupción tisular en situaciones de hipoxia ${ }^{(6)}$, isquemia ${ }^{(2,7)}$, sepsis ${ }^{(27)}$ o enterocolitis necrotizante ${ }^{(6)}$. Durante este proceso isquémico e inflamatorio pueden activarse células de la microglía ${ }^{(12,19)}$ y astroglía, que producen radicales libres y mayor cantidad de citoquinas (IL- $\alpha$, IL $\beta$, IL-6 e IL-8) que dañan directamente los preoligodendrocitos ${ }^{(19)}$ e inducen apoptosis por la vía de las caspasas ${ }^{(6)}$. Al respecto, Volpe explica que esta vulnerabilidad de los preoligodendrocitos y las neuronas de la subplaca se desarrolla entre las 24 y 32 semanas de gestación ${ }^{(7,27)}$.

Dado que la CA se basa en criterios clínicos que pueden llevar a falsos positivos o negativos ${ }^{(3,5,24)}$, usamos un modelo de análisis que relaciona la $\mathrm{CAH}$ con el daño neurológico por edades. Sorprendentemente, el $42 \%$ de las placentas examinadas presentaron $\mathrm{CAH}$, frecuencia que se encuentra en el límite superior de los reportes nacionales e internacionales ${ }^{(3,28)}$. Si bien la mayoría de las placentas afectadas mostraban infiltración en estadio inicial ${ }^{(3,20)}$ cuando se afectaba el corion y el amnios simultáneamente, el riesgo de daño neurológico se duplicó o triplicó tanto en la primera semana de vida como entre los 7 y 30 días, con diferentes patrones de daño cerebral asociados, pocas veces descrito en la literatura.

Frecuentemente las lesiones cerebrales fueron de carácter hemorrágico, isquémico y de sustancia blanca. Excepto las meningitis, todas fueron identificadas por ecografía cerebral, método elegido por su gran accesibilidad y eficiencia para determinar el tipo y la extensión de la lesión cerebral ${ }^{(20)}$. Llamó la atención la frecuencia de VLE, tipo de lesión de sustancia blanca, relacionada con procesos inflamatorios, seguida de la HIV, tal como ya se ha descrito en nuestro medio ${ }^{(8)}$

El análisis ajustado, excluyendo los factores de confusión, por subtipos de CAH y de acuerdo con la edad permitió determinar la asociación con algunas lesiones cerebrales. Solo el subtipo de afección concurrente de corion y amnios se relacionó con riesgo. Para HIV el riesgo se duplicó durante la primera semana, posiblemente por la asociación del componente inflamatorio a la fragilidad capilar ${ }^{(29)}$, independientemente de otros factores ${ }^{(2)}$. Por el contrario, el riesgo para las lesiones de sustancia blanca tipo LPV y VLE se triplicó entre los 7 y 30 días de edad, posiblemente por los mecanismos inflamatorios e isquémicos descritos anteriormente ${ }^{(7,19)}$.

No obstante, a las 40 semanas de edad corregida no se encontró asociación alguna pese a haber controlado los efectos confusores de las lesiones precedentes y de las enfermedades sistémicas concurrentes. Consideramos que la hipoplasia cerebral y otras lesiones fueron efecto de los múltiples procesos combinados entre los que destaca la desnutrición, los trastornos metabólicos (hiploglicemia, hiperbilirrubinemia), la displasia broncopulmonar, la neumonía, las sepsis tardías y la enterocolitis necrotizante, entre los que se diluye el propio efecto inflamatorio de la $\mathrm{CAH}^{(7,20,26)}$. Otras lesiones cerebrales muy pequeñas o difusas, que hubieran mejorado el análisis no se identificaron por falta de resonancia magnética ${ }^{(29,31)}$.

En este estudio se ha puesto en evidencia que varios mecanismos inflamatorios concurren al daño cerebral. El principal fue la infección placentaria que derivó en sepsis, y no habiendo evidencia de que la meningitis haya tenido un rol fundamental en el daño cerebral, consideramos que el factor inflamatorio derivado de la propia enfermedad séptica sería el responsable del efecto lesivo a largo plazo ${ }^{(26,29)}$. Otros procesos infecciosos e inflamatorios, como la neumonía, la enterocolitis necrotizante, el ductus arterioso persistente y la enfermedad de membrana hialina, estuvieron asociados a mayor daño neurológico, sobre todo entre los 7 y los 30 días de vida ${ }^{(29)}$, lo que abre una serie de posibilidades de manejo y control de las lesiones cerebrales por edades, que requieren mayor análisis a futuro.

Pese a ello, estudios recientes han concluido que no existe evidencia suficiente que justifique la asociación entre la $\mathrm{CAH}$ y las lesiones cerebrales en el prematuro ${ }^{(30,32)}$. Solo el estudio de Pappas, et al. ${ }^{(29)}$ encontró correlación entre la HIV severa y la CAH. Con respecto a la LPV, tipo de lesión más conocida, ninguno de los estudios posteriores al 2005 volvió a encontrar asociación. No obstante, la hipótesis permanece en discusión porque los estudios clínicos difieren en metodología y contrariamente existe buen sustento experimental. Shi, et al. ${ }^{(21)}$, sobre la base de una revisión sistemática y metaanálisis, recalcan que la CAH se asocia con riesgo de parálisis cerebral cuando los estudios son prospectivos y parten de la presencia o ausencia de corioamnionitis. Otros hacen énfasis en analizar el compromiso del amnios ${ }^{(32)}$. Sobre los efectos a largo plazo, Ylijoki, et al. demostraron que la $\mathrm{CAH}$ se encontraba asociada con menor desempeño cognitivo, de memoria y habilidades de aprendizaje a los 5 años ${ }^{(26)}$.

Particular interés fueron los efectos sobre los prematuros de menor edad gestacional, neonatos con mayor frecuencia y extensión de las lesiones cerebrales, a expensas del daño de la sustancia blanca, y que probablemente ocasiona un menor tamaño craneal. Estos hallazgos han sido descritos desde hace mucho ${ }^{(7,19)}$ y generalmente traducen severas discapacidades en la infancia temprana ${ }^{(16)}$. Sin embargo, existen muchos factores relacionados, en los que no ha quedado claramente definido el rol de la $\mathrm{CAH}^{(23,26)}$.

De acuerdo con los conocimientos actuales, los futuros proyectos deberán tener en cuenta los tipos (agudos y crónicos) y grados de CA, los gérmenes, el clampaje del cordón umbilical, la respuesta inflamatoria fetal (funisitis y amnios), la respuesta inflamatoria materna, la circulación de citoquinas deletéreas y protectoras, el uso de corticoides prenatales y posnatales, el seguimiento del perímetro cefálico, los hallazgos de resonancia magnética y los exámenes del neurodesarrollo ${ }^{(3,6,30)}$.

Como fortalezas se debe mencionar que se contó con los exámenes anatomopatológicos de la placenta, y los diagnós- 
ticos neurológicos fueron realizados por neuropediatras entrenados en ecografía cerebral. Asimismo, se realizó un modelo de análisis por edad, entendiendo la importancia de la fisiopatología de las lesiones cerebrales del prematuro, y un análisis multivariante que permitió considerar factores de confusión. Entre las limitaciones se debe mencionar que no se registró el tiempo de clampaje de cordón umbilical. Tampoco se diferenció la sepsis tardía que podría ser una variable de confusión en el efecto a las 40 semanas, y no se obtuvo cultivo de membranas o cultivo de líquido amniótico que podrían explicar mejor el factor de virulencia del germen.

En conclusión, la CAH se encontró en casi la mitad de los nacimientos de prematuros menores de 34 semanas. La ruptura prematura de membranas fue la principal generatriz de sepsis neonatal, y a su vez la sepsis se relacionó con el daño neurológico. La corioamnionitis generó daño cerebral en los prematuros de 0 a 7 días y de 7 a 30 días de edad. Las lesiones más frecuentes fueron HIV y daño de la sustancia blanca (VLE y LPV). A las 40 semanas de edad corregida, los prematuros extremos con $\mathrm{CAH}$ tuvieron lesiones cerebrales más extensas.

Agradecimiento: Al Dr. Rodrigo Carrillo por la edición y aportes para el desarrollo del manuscrito a través de la promoción de publicación de trabajos de investigación de la Facultad de Medicina Alberto Hurtado de la Universidad Peruana Cayetano Heredia. A los médicos asistentes, residentes, internos de medicina y licenciadas obstetras de los servicios de Neonatología y Obstetricia del Hospital Cayetano Heredia.

Contribuciones de los autores: DGP, NG y CLl participaron en la concepción, diseño y análisis de datos del estudio. NG, CLl participaron en el enrolamiento y recolección de datos. JV, SS y TJ participaron en la recolección de datos. DGP y NG participaron en la redacción, JC participó en la recolección de datos anatomopatológicos. Todos los autores revisaron y aprobaron el artículo.

Financiamiento: Investigación autofinanciada.

Conflicto de intereses: Los autores declaran no tener conflictos de interés.

\section{REFERENCIAS BIBLIOGRÁFICAS}

1. Eastman NJ, DeLeon M. The etiology of cerebral palsy. Am J Obstet Gynecol. 1955; 69:950-61. doi: 10.1016/0002-9378(55)90094-6.

2. Kaukola T, Herva R, Perhomaa M, Paakko E. Population Cohort Associating Chorioamnionitis, Cord Inflammatory Cytokines and Neurologic Outcome in Very Preterm, Extremely Low Birth Weight Infants. Pediatr Res. 2006;59:478-83.

3. Kim C, Romero R, Chaemsaithong P, Chaiyasit N, Yoon B, Kim Y Acute chorioamnionitis and funisitis: definition, pathologic features, and clinical significance. Am J Obstet Gynecol. 2015;213(4):S29-S52. doi: 10.1016/j.ajog.2015.08.040.

4. Ministerio de Salud. Análisis de la situación de salud de la provincia de Lima 2011 [Internet]. Lima: Dirección General de Epidemiologia, MINSA; 2011. Disponible en: http://bvs.minsa.gob.pe/local\%20/ MINSA/1778.pdf.

5. Edwards RK. Corioamnionitis y parto. Clinic Obstet Ginecol Norteam. 2005;32:287-96.

6. McAdams RM, Juul SE. The Role of Cytokines and Inflammatory Cells in Perinatal Brain Injury. Neurol Res Int. 2012:1-15. doi: $10.1155 / 2012 / 561494$.

7. Volpe JJ. Neurology of the Newborn. Sixth Edition. Elsevier Health Ed; 2018.

8. Guillén D. Diagnóstico de las Lesiones Cerebrales de los Prematuros Menores de 34 semanas: Incidencia, Factores asociados y Pronóstico [tesis doctoral]. Lima: Universidad Peruana Cayetano Heredia; 2004.

9. Gaudet LM, Smith GN. Cerebral Palsy and chorioamnionitis: The inflammatory cytokine link. Obstet Gynecol Surve. 2001;56(7):433-36.

10. Graham EM, Holcroft CJ, Rai KK, Donohue PK, Allen MC. Neonatal cerebral white matter injury in preterm infants is associated with culture positive infections and only rarely with metabolic acidosis. Am J Obstet Gynecol. 2004;191:1305-10.

11. Hagberg H, Mallard C, Ferriero DM, Vannucci SJ, Levison SW, Vexler $\mathrm{ZS}$, et al. The role of inflammation in perinatal brain injury. Nat Rev Neurol. 2015;11(4):192-208. doi: 10.1038/nrneurol.2015.13.

12. Dammann O, Leviton A. Infection remote from the brain, neonatal white matter damage, and cerebral palsy in the preterm infant. Seminar Pediatr Neurol. 1998;5(3):190-201.

13. Harry GJ, Lawler C, Brunseen SH. Maternal infection and white matter toxicity. Neurotoxicology. 2006; 27: 658-70.
14. Chau V, Poskitt KJ, McFadden D, Bowen-Roberts T, Synnes A, Brant R. Effect of Chorioamnionitis on Brain Development and Injury in Premature Newborns. Ann Neurol. 2009; 66:155-64. doi: 10.1002/ana.21713.

15. Ylijoki M, Ekholm E, Haataja L, Lehtonen L. Is chorioamnionitis harmful for the brain of preterm infants? A clinical overview. Acta Obstet Gynecol Scand. 2012;91(4):403-19. doi: 10.1111/j.1600-0412.2012.01349.x.

16. Medina-Alva P, Duque KR, Zea-Vera A, Bellomo S, Cárcamo C, Guillén-Pinto $\mathrm{D}$, et al. Combined predictors of neurodevelopment in very low birth weight preterm infants. Early Hum Dev. 2019;130:109-15. doi: 10.1016/j.earlhumdev.2019.01.019.

17. Zea-Vera A, Turin CG, Ochoa TJ. Unificar los criterios de sepsis neonatal tardía: propuesta de un algoritmo de vigilancia diagnóstica. Rev Peru Med Exp Salud Publica. 2014;31(2):358-63.

18. Norma técnica para la atención integral de salud neonatal. Resolución $\mathrm{N}^{\circ}$ 828-2013/MINSA [Internet]. Ministerio de Salud; 2015. Disponible en: http://bvs.minsa.gob.pe/local/minsa/3281.pdf.

19. Volpe JJ. Brain Injury in premature infants: a complex amalgam of destructive and developmental disturbances. Lancet Neurol. 2009; 8:110-24. doi: 10.1016/S1474-4422(08)70294-1.

20. Maisonneuve E, Ancel P, Foix-L'Hélias L, Marret S, Kayem G. Impact of clinical and/or histological chorioamnionitis on neurodevelopmental outcomes in preterm infants: A literature review. J Gynecol Obstet Hum Reprod. 2017;46(4):307-316. doi: 10.1016/j.jogoh.2017.02.007.

21. Shi Z, Ma L, Luo K, Bajaj M, Chawla S, Natarajan G, et al. Chorioamnionitis in the Development of Cerebral Palsy: A Meta-analysis and Systematic Review. Pediatrics 2017;139(6):e20163781.doi: 10.1542/peds.2016-3781.

22. Rincón I, Magdaleno F, Sancha N, Omeñaca F, González A. Corioamnionitis histológica y morbimortalidad neonatal: Aproximación al síndrome de respuesta inflamatoria fetal. Rev Chil Obstet Ginecol. 2010;75(3):172-8.

23. Pugni L, Pietrasanta C, Acaia B, Merlo D, Ronchi A, Ossola M, et al. Chorioamnionitis and neonatal outcome in preterm infants: a clinical overview. J Matern Fetal Neonatal Med. 2016;29(9):1525-29. doi: 10.3109/14767058.2015.1053862.

24. Chaiyasit N, Romero R, Chaemsaithong P, Docheva N, Bhatti G, Kusanovic JP, et al. Clinical chorioamnionitis at term VIII: a rapid MMP-8 test for the identification of intra-amniotic inflammation. J Perinat Med. 2017: 26;45(5):539-550. doi: 10.1515/jpm-2016-0344. 
25. Amiya RM, Mlunde LB, Ota E, Swa T, Oladapo OT, Mori R. Antenatal Corticosteroids for Reducing Adverse Maternal and Child Outcomes in Special Populations of Women at Risk of Imminent Preterm Birth: A Systematic Review and Meta-Analysis. PLoS One. 2016;11(2):e0147604. doi: 10.1371/journal.pone.0147604.

26. Ylijoki M, Lehtonen L, Lind A, Ekholm E, Lapinleimu H, Kujari H, et al. Chorioamnionitis and Five-Year Neurodevelopmental Outcome in Preterm Infants. Neonatology. 2016;110(4):286-295.

27. Khwaja O, Volpe JJ. Pathogenesis of Cerebral White matter injury of prematurity. Arch Dis Child Fetal Neonatal Ed. 2008;2:153-61. doi: 10.1136/ adc.2006.108837.

28. Instituto Nacional de Estadística e Informática. Encuesta Demográfica y de Salud Familiar 2015 [Internet]. Lima: INEI;2015. Disponible en: https://www.
inei.gob.pe/media/MenuRecursivo/publicaciones_digitales/Est/Lib1356/.

29. Pappas A, Kendrick D, Shankaran S, Stoll B, Bell E, Laptook A, et al. Chorioamnionitis and Early Childhood Outcomes Among Extremely Low-Gestational-Age Neonates. JAMA Pediatrics. 2014;168(2):137-47.

30. Chau V, McFadden D, Poskitt KJ, Miller SP. Chorioamnionitis in the Pathogenesis of Brain Injury in Preterm Infants. Clin Perinatol. 2014;41:83103. doi: 10.1016/j.clp.2013.10.009.

31. Anblagan D, Pataky R, Evans MJ, Telford EJ, Serag A, Sparrow S, et al. Association between preterm brain injury and exposure to choriamnionitis during fetal life. Sci Rep. 2016;6:37932. doi: 10.1038/srep37932.

32. Rocha G, Proenca E, Quintas C, Rodrigues T, Guimaraes H. Chorioamnionitis and brain damage in preterm Newborn. J Matern Fetal Neonatal Med. 2007;20(10):745-9. doi: 10.1080/14767050701580515. 